H. Opolka

Nagoya Math. J.

Vol. 92 (1983), 179-186

\title{
EXTENSIONS OF NUMBER FIELDS DEFINED BY COHOMOLOGY GROUPS
}

\author{
HANS OPOLKA
}

\section{§1. Introduction}

Let $k$ be a field of characteristic 0 , let $\bar{k}$ be an algebraic closure of $k$ and denote by $G_{k}=G(\vec{k} / k)$ the absolute Galois group of $k$. Suppose that for some natural number $n \geq 3$ the cohomology group $H^{n}\left(G_{k}, Z\right)$ is trivial. Then, given any finite factor group $G=G(K / k)$ of $G_{k}$, there is for every subgroup $\mathscr{A} \leq H^{n}(G, Z)$ a finite Galois extension $L$ of $k$ containing $K, L \subset \bar{k}$, such that $\mathscr{A}$ becomes trivial under inflation inf: $H^{n}(G, Z)$ $\rightarrow H^{n}(G(L / k), Z)$. Any finite Galois extension $L$ of $K / k$ with this property is called an extension of $K / k$ defined by $\mathscr{A}$. One purpose of this note is to show that in case $k$ is a global or local number field every $\mathscr{A}$ defines an extension $L$ of $K / k$ which is abelian over $K$. It seems to be rather difficult to get precise information about these extensions. For $n=3$ the situation is quite well understood: it corresponds to genus theory and the theory of central extensions. Some informations are provided for $n \geq 4$ in case $K / k$ is cyclic.

\section{§2. Notations}

$k$ a global or local number field, i.e. a finite extension of $\boldsymbol{Q}$ or a finite extension of $\boldsymbol{Q}_{p}, \boldsymbol{R}$ or $=\boldsymbol{C}$

$k^{*} \quad$ the multiplicative group of $k$

$\bar{k} \quad$ an algebraic closure of $k$

$G_{k}=G(\bar{k} / k)$ the absolute Galois group of $k$

$G \quad=G(K / k)$ a finite factor group of $G_{k}$

$I_{K} \quad=$ idele group, if $K$ is global

$C_{K} \quad=$ idele class group of $K$ if $K$ is global, =the multiplicative group $K^{*}$ if $K$ is local

$D_{K} \quad=$ the universal norm subgroup of $C_{K}$ 


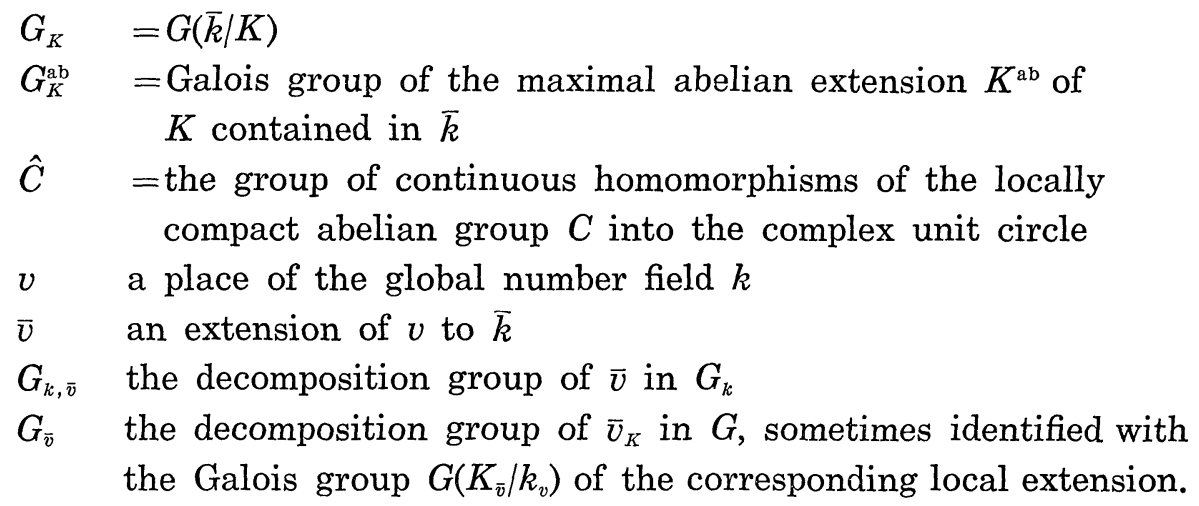

\section{§3. Strict cohomological dimension}

Our starting point is the following well known theorem, see e.g. [9], II, in connection with [10], Section 6.

(3.1) Theorem. Suppose $k$ is local. Then

$$
\begin{aligned}
& H^{2 n+1}\left(G_{k}, Z\right)=0, \quad n \geq 1 \\
& H^{2 n}\left(G_{k}, Z\right) \cong\left\{\begin{array}{ll}
0, & \text { if } k \neq \boldsymbol{R} \\
Z / 2, & \text { if } k=\boldsymbol{R}
\end{array}, \quad n \geq 2 .\right.
\end{aligned}
$$

Suppose $k$ is global. Then

$$
\begin{aligned}
& H^{2 n+1}\left(G_{k}, Z\right)=0, \quad n \geq 1 \\
& H^{2 n}\left(G_{k}, Z\right)=(Z / 2)^{r_{1}}, \quad r_{1}=\text { number of real places of } k, n \geq 2 .
\end{aligned}
$$

(3.2) Corollary. Suppose $G=G(K / k)$ is a finite factor group of $G_{k}$. Then a subgroup $\mathscr{A} \leq H^{n}(G, Z), n \geq 3$, defines a finite Galois extension of $K / k$ in the sense of Section 1 in the following cases:

(a) $k$ is a global or local number field and $n$ is odd $\geq 3$

(b) $k$ is local $\neq \boldsymbol{R}$ and $n$ is even $\geq 4$

(c) $k$ is global and totally imaginary and $n$ is even $\geq 4$.

A pair $(k, n)$, consisting of a global or local number field $k$ and $a$ natural number $n \geq 3$, is called ordinary, if one of the conditions (a), (b) or (c) of (3.2) holds.

\section{§4. Abelian extensions defined by cohomology}

In this section we shall prove the following result.

(4.1) Theorem. Suppose $(k, n)$ is an ordinary pair in the sense of 
Section 3 and $G=G(K / k)$ is a finite factor group of $G_{k}$. Then every subgroup $\mathscr{A} \leq H^{n}(G, Z)$ defines a finite Galois extension $L$ of $K / k$ such that $L / K$ is abelian. Furthermore, for $n=3$ we can choose $L$ in such a way that $G(L / K) \subset$ center $(G(L / k))$.

For the proof we shall use some more notation. Denote by $u_{0} \in$ $H^{2}\left(G, C_{K}\right)$ the canonical class. As is well known, the universal Artin map [1]

$$
\alpha: C_{K} \longrightarrow G_{K}^{\mathrm{ab}}
$$

is $G$-equivariant. Hence $\alpha$ induces a map on cohomology groups

$$
\alpha^{*}: H^{2}\left(G, C_{K}\right) \longrightarrow H^{2}\left(G, G_{K}^{\mathrm{ab}}\right) .
$$

Set

$$
u=\alpha^{*}\left(u_{0}\right)
$$

The group extension defined by $u$ yields a transgression map

$$
\tau=\tau_{3}: \hat{G}_{K}^{G} \longrightarrow H^{2}(G, \boldsymbol{Q} \mid Z) \cong H^{3}(G, Z)
$$

which is given explicitly as follows

$$
\tau(\chi)(\delta, \rho)=\chi(u(\delta, \rho)), \quad \chi \in \hat{G}_{K}^{G}, \quad \delta, \rho \in G .
$$

This map is part of the Hochschild-Serre exact sequence, [11], II, Section 4,

$$
1 \longrightarrow \hat{G} \stackrel{\text { inf }}{\longrightarrow} \hat{G}_{k} \stackrel{\text { res }}{\longrightarrow} \hat{G}_{K}^{G} \stackrel{\tau}{\longrightarrow} H^{2}(G, \boldsymbol{Q} / Z) \longrightarrow 0
$$

$\tau$ is surjective because $H^{2}\left(G_{k}, \boldsymbol{Q} / Z\right) \cong H^{3}\left(G_{k}, Z\right)=0$.

Proof of (4.1) for $n=3$. Take a finite subset $X \subset \hat{G}_{k}^{G}$ such that $\tau(X)$ $=\mathscr{A}$ and define $L$ to be the fixed field of the intersection of all $\operatorname{Ker}(\chi)$, $\chi \in X$. Since $X$ is finite and since every $\chi \in X$ is invariant under the action of $G, L$ is a finite central extension of $K / k$. The Hochschild-Serre exact sequence (4.2) shows that $\mathscr{A}$ becomes trivial under inflation $H^{3}(G, Z) \rightarrow H^{3}(G(L / k), Z)$.

In order to prove (4.1) for $n \geq 4$ we need some more cohomology. Suppose $g$ is a profinite group such that its strict cohomological dimension is 2. Take open subgroups $V, W \leq \mathfrak{g}$ such that $V$ is normal in $W$. Then there is a spectral-sequence [11], II, Section 4,

$$
E_{2}^{p, q}=H^{p}\left(W / V, H^{q}(V, \boldsymbol{Q} / Z)\right) \Longrightarrow H^{r}(W, \boldsymbol{Q} / Z) \text {. }
$$


By assumption $E_{2}^{p, q}$ is trivial for $q \neq 0,1$. Hence the spectral sequence yields the following exact sequence

$$
\cdots \longrightarrow H^{i}(W, \boldsymbol{Q} / Z) \longrightarrow E_{2}^{i-1,1} \stackrel{d_{2}}{\longrightarrow} E_{2}^{i+1,0} \longrightarrow H^{i+1}(W, \boldsymbol{Q} / Z) .
$$

So for $i \geq 2$ there is an isomorphism

$$
d_{2}: H^{i-1}(W / V, \hat{V}) \stackrel{\sim}{\longrightarrow} H^{i+1}(W / V, \boldsymbol{Q} / Z),
$$

which is obtained by cup product with the cocycle class corresponding to the group extension $1 \rightarrow V / V^{\prime} \rightarrow W / V^{\prime} \rightarrow W / V \rightarrow 1$.

Now suppose $(k, n)$ is an ordinary pair in the sense of Section 3 and $n \geq 4$. Then (4.3) with $\mathfrak{g}=W=G_{k}, V=G_{K}$ yields an isomorphism

$$
\tau_{n}: H^{n-3}\left(G, \hat{G}_{K}\right) \longrightarrow H^{n-1}(G, Q / Z) \cong H^{n}(G, Z), \quad n \geq 4,
$$

Proof of (4.1) for $n \geq 4$. For any finite Galois extension $L$ of $K / k$, $L \subset \bar{k}$, with Galois group $\tilde{G}=G(L / k)$ there is a homomorphism

$$
\rho_{n}: H^{n-3}\left(G, \hat{G}_{K}\right) \longrightarrow H^{n-3}\left(\tilde{G}, \hat{G}_{L}\right)
$$

such that the following diagram commutes

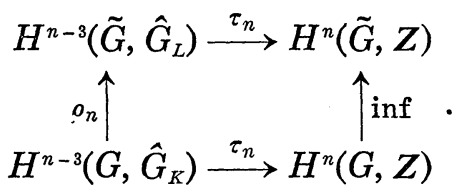

Explicitly $\rho_{n}$ is given as follows

$$
\rho_{n}(\lambda)\left(y_{1}, \cdots, y_{n-3}\right)(x)=\lambda\left(\bar{y}_{1}, \cdots, \bar{y}_{n-3}\right)(x)
$$

for all $(n-3)$-cocycles $\lambda$ on $G$ with values in $\hat{G}_{K}$, all $\left(y_{1}, \cdots, y_{n-3}\right) \in \tilde{G}^{(n-3)}$ and all $x \in G_{L} ; \bar{y}_{i}$ denotes the image of $y_{i}$ under the natural map $\tilde{G} \longrightarrow G$. Now suppose $\bar{\lambda} \in \tau_{n}^{-1}(\mathscr{A})$. Define $X$ to be the set of all characters $\lambda^{\delta}\left(x_{1}, \cdots, x_{n-3}\right) \in \hat{G}_{K},\left(x_{1}, \cdots, x_{n-3}\right) \in G^{(n-3)}, \delta \in G$, and let $L$ denote the fixed field corresponding to the intersection of all $\operatorname{Ker}(\chi), \chi \in X$. Then $L$ is a finite Galois extension of $K / k$, abelian over $K$, and $\bar{\lambda}$ becomes trivial under $\rho_{n}$. Therefore $\tau_{n}(\bar{\lambda})=\bar{f} \in \mathscr{A}$ becomes trivial under inflation. Apply this construction to all $\bar{f} \in \mathscr{A}$ and form the composition of all $L$ obtained in this way. This field is a finite Galois extension of $K / k$, abelian over $K$, such that $\mathscr{A}$ becomes trivial under inflation. 


\section{§5. Some special cases}

The purpose of this section is to illustrate the general result (4.1) in the special case $n=3$ and, when the given field extension is cyclic, also in the case $n \geq 4$. The case $n=3$ leads to the theory of central extensions, to genus theory and to some questions centering around the Hasse norm theorem. There is an extensive literature dealing with these questions, see e.g. [3], [4], [7], [8], [12], [13] and also the references in these articles. So we shall confine ourselves with some brief remarks. Suppose $k$ is a global or local number field and $G=G(K / k)$ is a finite factor group of $G_{k}$. A finite Galois extension $L$ of $K / k$ is called central, if $G(L / K) \subset$ center $(G(L / k))$. Two central extensions $L_{1}, L_{2}$ of $K / k$ are said to belong to the same genus if there is a finite abelian extension $k_{0} / k$, $k_{0} \subset \bar{k}$, such that $L_{1} \cdot k_{0}=L_{2} \cdot k_{0}$. By (4.1) every subgroup $\mathscr{A} \leq H^{3}(G, Z)$ defines a set $M(\mathscr{A})$ of central extensions of $K / k$. Using the HochschildSerre sequence (4.2) one observes

(5.1) The assignment $\mathscr{A} \mapsto M(\mathscr{A})$ establishes a bijective correspondence between the set of subgroups of $H^{3}(G, Z)$ and the set of genera of central extensions of $K / k$. Furthermore, for every $L \in M(\mathscr{A})$ the transgression map yields an epimorphism $G(L / K)^{\wedge} \rightarrow \mathscr{A}$.

Given a subgroup $\mathscr{A} \leq H^{3}(G, Z)$ one can ask for canonical representatives of $M(\mathscr{A})$, for instance, one can try to find representatives with small degree or "small" conductor. As far as I know there are no complete answers to these questions in the literature. It seems obvious that complete solutions to these problems and further precise arithmetical interpretations of the cohomology group $H^{3}(G(K / k), Z)$ for finite $p$-extensions $K / k$ will help to get deeper insight in the theory of nilpotent extensions of number fields.

In order to study local-global questions one introduces in the global situation the kernel $\mathscr{H}=\mathscr{H}(K / k)$ of the localization map

$$
H^{3}(G, Z) \longrightarrow \coprod_{v} H^{3}\left(G_{\bar{v}}, Z\right) \text {. }
$$

As Tate observes [2], p. 198, $\mathscr{H}$ has the following arithmetical interpretation.

(5.2) $\mathscr{H}$ is dual to the obstruction group $\mathscr{K}=\mathscr{K}(K / k)$ for the Hasse norm principle, i.e. $\mathscr{H}$ is dual to $\mathscr{K}=\left\{a \in k^{*} \mid a\right.$ is a norm locally everywhere in $K / k\}$ modulo $\operatorname{Norm}_{K / k}\left(K^{*}\right)$. 
This observation has been the starting point for investigations which try to classify extensions of number fields for which the Hasse norm principle holds, see e.g. [7]. It is amusing to dualize the statemant " $\mathscr{H}$ defines the central extension $L$ of $K / k$ ". It reads as follows, see [6].

(5.3) Suppose $a \in k^{*}$ is a norm locally everywhere in $L / k$. Then $a$ is a global norm in $K / k$.

Now suppose $k$ is a global or local number field and $(k, n), n \geq 4$, is an ordinary pair in the sense of Section 3. In the following we assume that $K / k$ is cyclic and try to give some information about extensions of $K / k$ which are defined by $H^{n}(G, Z)$. We shall prove

(5.4) Theorem. Suppose $K / k$ is cyclic. Then every finite Galois extension $L$ of $K / k$ which is defined by $H^{n}(G(K / k), Z), n \geq 4$, corresponds to a G-invariant norm subgroup $A \leq C_{K}$ with the property $A \cap C_{k} \leq$ Norm $_{K / k} C_{K}$ and contains an obelian extension $K_{0} / k$ such that $K: k$ divides $K_{0}: K$.

Proof. For cyclic groups cohomology is periodic mod 2, so we have

$$
H^{n}(G, Z) \cong \begin{cases}0, & \text { if } n \text { is odd } \\ \hat{G}, & \text { if } n \text { is even }\end{cases}
$$

and

$$
H^{n-3}\left(G, \hat{G}_{K}\right) \cong \begin{cases}0, & \text { if } n \text { is odd } \\ H^{-1}\left(G, \hat{G}_{K}\right), & \text { if } n \text { is even }\end{cases}
$$

The Artin map induces isomorphisms $\hat{G} \cong H^{0}\left(G, C_{K}\right)^{\wedge}$ and $H^{-1}\left(G, \hat{G}_{K}\right)$ $\cong H^{-1}\left(G,\left(C_{K} / D_{K}\right)^{\wedge}\right)$. The isomorphism (4.4) induces an isomorphism $H^{-1}\left(G, \hat{G}_{K}\right) \cong \hat{G}$. Moreover, the restriction map induces an isomorphism res: $H^{-1}\left(G,\left(C_{K} / D_{K}\right)^{\wedge}\right) \rightarrow H^{0}\left(G, C_{K}\right)^{\wedge}$. A tedious but straightforward computation shows that all maps fit together in the following commutative diagram

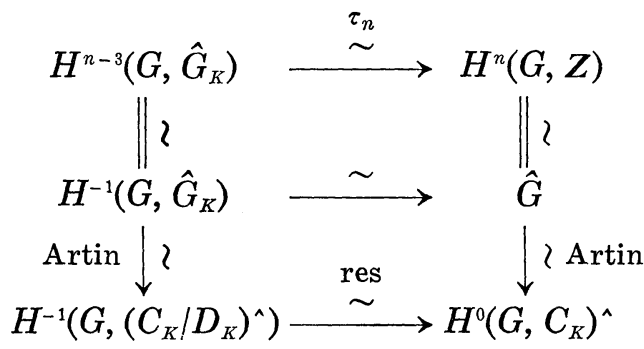


Now suppose that $L$ is a finite Galois extension of $K / k$, abelian over $K$, which is defined by $H^{n}(G, Z), n \geq 4$, see (4.1). Then $L$ defines a $G$-invariant norm subgroup $A \leq C_{K}$. Denote by $(\chi)$ a generator of $H^{-1}\left(G,\left(C_{K} / D_{K}\right)^{\wedge}\right)$. The above diagram shows that $A \leq \operatorname{Ker}(\chi)$ for some representative $\chi$ of $(\chi)$. Furthermore we have $\operatorname{Ker}(\chi) \cap C_{k}=\operatorname{Ker}\left(\chi_{C_{k}}\right)=$ Norm $_{K / k} C_{K}$, hence $A \cap C_{k} \leq \operatorname{Norm}_{K / k} C_{K}$. Now we shall use the following group theoretic version of Hilbert's theorem 94 which is due to $\mathrm{K}$. Miyake*).

(5.5) Suppose $H$ is a finite group with abelian normal subgroup $N \unlhd H$ and cyclic factor group $G=H / N$. Then $|G|$ divides $\operatorname{Ker}$ (transfer: $H \rightarrow N):[H, H]$

In the above situation set $H=G\left(L_{\chi} / k\right)$, where $L_{\chi}$ is the normal closure of the extension of $k$ corresponding to $\operatorname{Ker}(\chi) \leq C_{K}$, and $N=$ $G\left(L_{x} / K\right)$. It is well known that the following diagram commutes

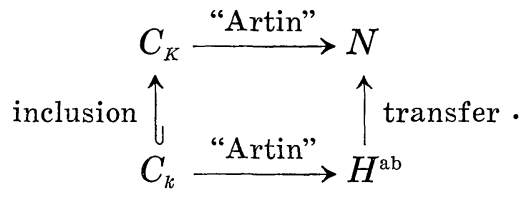

The equation $\operatorname{Ker}(\chi) \cap C_{k}=\operatorname{Norm}_{K / k} C_{K}$ implies Ker (transfer: $H \rightarrow N$ ) $\leq N$. Therefore (5.5) shows that $K: k$ divides $L_{\chi}^{\prime}: K$ where $L_{x}^{\prime}$ is the maximal abelian extension of $k$ contained in $L_{x}$. This completes the proof of (5.4).

\section{REFERENCES}

[1] E. Artin and J. Tate, Class Field Theory, Benjamin, New York, 1967.

[ 2 ] J.W.S. Cassels and A. Fröhlich, Algebraic number theory, Academic Press, New York, 1967.

[ 3 ] A. Fröhlich, On fields of class two, Proc. London Math. Soc., 4 (1954), 235-256.

[4] K. Masuda, An application of the generalized norm residue symbol, Proc. Amer. Math. Soc., 10 (1959), 245-252.

[5] K. Miyake, On the structure of the idele groups of algebraic number fields II, Tohoku Math. J., 34 (1982), 101-112.

[6] H. Opolka, Zur Auflösung zahlentheoretischer Knoten, Math. Z., 173 (1980), 95103.

[ 7 ] M. J. Razar, Central and genus class fields and the Hasse norm theorem, Compositio Math., 35 (1977), 281-298.

*) I would like to thank K. Miyake for an interesting correspondence and for communicating to me his result (5.5). 
[ 8 ] A. Scholz, Totale Normenreste, die keine Normen sind, als Erzeuger nichtabelscher Körpererweiterungen II, J. Reine Angew. Math., 182 (1940), 217-234.

[ 9 ] J. P. Serre, Cohomologie Galoisienne, Lecture Notes in Math., 5 Springer (1965).

[10] - Modular forms of weight one and Galois representations, in: Algebraic number fields (ed. A. Fröhlich), Durham Symposium, Academic Press, London, 1977.

[11] S. Shatz, Profinite groups, arithmetic and geometry, Princeton University Press, 1972.

[12] S. Shirai, On the central class field mood $\mathfrak{m}$ of Galois extensions of an algebraic number field, Nagoya Math. J., 71 (1978), 61-85.

[13] K. Miyake, On central extensions of a Galois extension of algebraic number fields, Manuscript, 1982.

Mathematisches Institut

Einsteinstrasse 64

D-4400 Münster 